\section{PWE-054 FLEXIBLE SIGMOIDOSCOPY PRIOR TO CTC WITHIN THE BCSP}

R Rusu*, G Smith, J Martin. Gastroenterology Department, Imperial College Healthcare NHS Trust, London, UK

\subsection{6/gutjnl-2014-307263.314}

Introduction Patients with abnormal FOBt results within the Bowel Cancer Screening Programme (BCSP) are at increased risk of colorectal neoplasia and are therefore offered colonoscopy. Some patients, with significant co-morbidities, are not suitable for colonoscopy and are offered CT colonography (CTC) as an alternative. There has been concern that the insufflation tube used during this examination may obscure visualisation of low rectal lesions and this has been reported in the literature. At the West London Bowel Cancer Screening Centre all patients are offered a flexible sigmoidoscopy (FS) prior to CTC and our experience of this approach is reported in this abstract.

Methods All patients with an abnormal FOBt result, attending a Specialist Screening Practitioner (SSP) clinic between $4^{\text {th }}$ January 2012 and $1^{\text {st }}$ October 2013, and who were offered CTC and FS were identified. Their endoscopic and radiologial investigations were retrieved from the hospital electronic records system and the results recorded in terms of the adenomas and cancers identified.

Results 1544 patients were seen in an SSP clinic within the allocated period, and $73(4.7 \%)$ of these were offered CTC. Of these $49(67.1 \%)$ had a FS as the first investigation and 24 (32.9\%) had a CTC as the first investigation. 10 (13.7\%) refused FS and 14 (19.2\%) had endoscopic investigations (12 FS and 2 colonoscopy) after the CTC, due to patient choice. 6 (8.2\%) patients who underwent FS as the first investigation had subsequent colonoscopy without CTC, 5 as a large polyp requiring resection was found at FS and 1 following detection of a cancer. In 3 of these patients further adenomas were found at colonoscopy. In total 67 patients $(91.8 \%)$ had CTC and in this group $12(17.9 \%)$ had a subsequent colonoscopy as a result of the radiological findings. Of these 6 had a normal FS prior to CTC and 6 patients did not have a FS. In 8 cases (66.7\%) the number of polyps seen on CTC was confirmed at colonoscopy, in 3 cases (25.0\%) more lesions were found at colonoscopy than CTC and in 1 case $(8.3 \%)$ CTC had identified more polyps than were seen at colonoscopy. 12 patients had FS after a normal CTC and no additional findings were seen on these examinations. No rectal lesions were identified at FS that were not seen at CTC. Overall 18 (24.7\%) patients eventually had a colonoscopy.

Conclusion FS prior to CTC within the BCSP does not appear to be of value in detecting additional rectal lesions missed at CTC, althou gh the numbers in this analysis are small. Initial FS avoids the need for CTC in about $8 \%$ of patients, and so should be performed before CTC, but this benefit needs to balanced against the increased workload and inconvenience to patients. In those patients in whom the initial decision is to perform CTC a quarter will eventually require a colonoscopy.

Disclosure of Interest None Declared.

\section{PWE-055 BOWEL CLEANSING AGENTS ENHANCE READER CONFIDENCE LEVELS WHEN EXCLUDING SIGNIFICANT FINDINGS AT CAPSULE ENDOSCOPY}

R Rameshshanker*, A O'Rourke, J Butcher, C Fraser. Wolfson Endoscopy Unit, St Mark's Hospital, London, UK
Introduction Capsule endoscopy (CE) is the first line of investigation for examining the small bowel (SB) mucosa. While standard preparation (SP) is more convenient for patients, mucosal visibility may deteriorate within the distal SB. Recent meta-analyses suggest that bowel-cleansing agents (BCA) can improve small bowel image quality (IQ). The influence of BCA compared to SP on reader confidence levels (RCL) when excluding clinically significant findings (CSF) has not been examined.

Methods

Aim

To compare RCL when excluding CSF and assessing IQ during reading following $\mathrm{SP}$ or $\mathrm{BCA}$ prior to $\mathrm{CE}$.

Methodology

We performed a retrospective analysis of SB capsule images of 100 consecutive patients who underwent a complete CE examination at our institution from Oct 2012 - Mar 2013. Patients had SP (intake of clear liquids for $18 \mathrm{~h}$ and $12 \mathrm{~h}$ fasting prior to the procedure without BCA) or BCA (2l of polyethylene glycol (PEG) or magnesium citrate (MC) in addition to SP). The participants' demographic and clinical data were collected and SB transit time (SBTT) calculated. A four point scale was used to assess IQ (grade $1=<80 \%$ of mucosa visible \pm excessive debris \pm severely reduced brightness to grade $4=\geq 90 \%$ of mucosa visible \pm mild debris \pm mildly reduced brightness). The SBTT was divided into quartiles (Q1-Q4) by time and the IQ score, RCL and number of CSF for each quartile were determined by a gastroenterologist experienced in $\mathrm{CE}$, blinded to the preparation. Procedures were examined in randomised order.

Results 49 (49\%) patients had SP (group A) while 51 (51\%) had one of the BCA (39\% had PEG and 61\% had MC, group B). There was no significant difference in age $(\mathrm{p}=0.87)$, sex $(\mathrm{p}=$ $0.57)$, indication $(\mathrm{p}=0.25)$ and SBTT (group A: $264 \pm 112$ mins vs. group $\mathrm{B}: 233 \pm 100$ mins, $\mathrm{p}=0.14$ ) between groups. For each quartile, IQ scores were significantly higher for group B than A except in Q1 (Q1: $3.7 \pm 0.7$ vs. $3.5 \pm 0.6, \mathrm{p}=0.06$; $\mathrm{Q} 2: 3.6 \pm 0.5$ vs. $3.1 \pm 0.6, \mathrm{p}<0.0001 ; \mathrm{Q} 3: 3.2 \pm 0.6$ vs. $2.3 \pm 0.7, \mathrm{p}<0.0001 ; \mathrm{Q} 4: 2.8 \pm 0.5$ vs. $1.9 \pm 0.8, \mathrm{p}<$ $0.0001)$. There was no difference in detection of CSF between group A and B ( $41 \%$ vs. $51 \%, \mathrm{p}=0.33$, respectively). For each quartile, RCL for excluding significant findings were significantly higher for group B than A except in Q1 (Q1: 100\% vs. 96\%, p $=0.06$; Q2: $96 \%$ vs. $73 \%, \mathrm{p}<0.0001$; Q3: $88 \%$ vs. $33 \%, \mathrm{p}<$ 0.0001; Q4: $77 \%$ vs. $20 \%, \mathrm{p}<0.0001)$. There was no significant difference in IQ or RCL between PEG or MC. 3 procedures (all SP) were considered unsatisfactory for IQ with recommendation to repeat these after BCA.

Conclusion BCA pre-capsule endoscopy significantly improve small bowel IQ and RCL when excluding CSF. Bowel cleansing appears to be an important parameter for optimising the qualitive aspects of CE reading.

Disclosure of Interest None Declared.

\section{PWE-056 DELIVERY OF BOWELSCOPE SCREENING - EXPERIENCE FROM THE PILOT SITES}

\footnotetext{
${ }^{1,2} \mathrm{R}$ Bevan* ${ }^{3}{ }^{3}$ P Patnick, ${ }^{4} \mathrm{R}$ Loke, ${ }^{5} \mathrm{~B}$ Saunders, ${ }^{6} \mathrm{~J}$ Stebbing, ${ }^{7} \mathrm{R}$ Tighe, ${ }^{8} \mathrm{~A}$ Veitch, ${ }^{1} \mathrm{~J}$ Painter, ${ }^{1,9,10} \mathrm{CJ}$ Rees. ${ }^{1}$ South of Tyne and Wear BCSC, Gateshead, UK; ${ }^{2}$ Northern Region Endoscopy Group, Newcastle, UK; ${ }^{3}$ NHS Cancer Screening Programmes, Sheffield, UK; ${ }^{4}$ West Kent and Medway BCSC, Tunbridge Wells, UK; ${ }^{5}$ St Marks BCSC, London, UK; ${ }^{6}$ Surrey BCSC, Guilford, UK; ${ }^{7}$ Norwich BCSC, Norwich, UK; ${ }^{8}$ Wolverhampton BCSC, Wolverhampton, UK; ${ }^{9}$ Northern Region Endoscopy Group, Newcastle Upon Tyne, UK;

${ }^{10}$ Durham University, Stockton on Tees, UK
} 
BSG 2014 abstracts

\begin{tabular}{|c|c|c|c|c|c|c|c|c|c|}
\hline \multirow[b]{2}{*}{ Centre } & \multirow[b]{2}{*}{ First list } & \multirow{2}{*}{$\begin{array}{l}\text { Endoscopists } \\
\text { (BCSP colonoscopists) }\end{array}$} & \multicolumn{4}{|c|}{ Endoscopist grade } & \multirow[b]{2}{*}{ Lists per week } & \multirow[b]{2}{*}{ Evenings/ Weekends } & \multirow[b]{2}{*}{ SSPs per list } \\
\hline & & & Consultant & Registrar & Staff/ other grade & Nurse endoscopist & & & \\
\hline Norwich & 08.07 .13 & $6(1)$ & 0 & 3 & 0 & 3 & 6 & Yes/Yes & $1^{*}$ \\
\hline South of Tyne & 07.05 .13 & $2(0)$ & 0 & 1 & 1 & 0 & 2.5 & Yes/Yes & 2 \\
\hline St Marks & 22.07 .13 & $8(2)$ & 1 & 5 & 0 & 2 & 7 & Yes/No & $1^{*}$ \\
\hline Surrey & 07.11 .13 & $6(6)$ & 6 & 0 & 0 & 0 & 2 & No/Yes & 2 \\
\hline West Kent and Medway & 11.06 .13 & 7 (3) & 1 & 0 & 5 & 1 & 4 & No/Yes & 2 \\
\hline W'hampton & 07.08 .13 & $6(3)$ & 4 & 0 & 2 & 0 & 6 & No/No & 1 \\
\hline
\end{tabular}

Introduction The NHS Bowel Cancer Screening Programme (BCSP) is being expanded to include a single flexible sigmoidoscopy (FSIG) called BowelScope, offered to all 55 year olds in addition to biannual faecal occult blood testing from age 60-75 years.

6 pilot sites began BowelScope screening in May 2013, with a view to full English coverage by the end of 2016 .

Methods We aim to describe practical issues involved in the delivery of BowelScope screening at the pilot centres, covering unit set-up, list format, and endoscopists delivering lists.

A survey was sent to the 6 pilots for completion by screening staff. Data were also retrieved from the national BCSP database.

Results The first BowelScope list was delivered in the South of Tyne Screening Centre on $7^{\text {th }}$ May 2013. By December 2013, 4135 flexible sigmoidoscopy procedures had been performed in 6 centres.

Centres have delivered 20-80 lists each, performing 2-7 lists per week. Sessions are run at varying times of day including evenings and Saturdays.

35 endoscopists undertake lists regularly, of whom 15 were already BCSP accredited. Other BCSP colonoscopists provide back up for lists when required. All non-BCSP were accredited through a combination of direct observation of procedural skills (DOPS) and an MQC exam.

Specialist Screening Practitioners (SSPs) attend all lists, and are deployed in a variety of ways including: following patient journey, consenting or giving information, and supervision assistant SSPs.

Table 1 shows details of screening lists by centre.

Conclusion BowelScope screening is being successfully delivered at the six pilot centres. Each centre has developed a screening template and organisational pattern that works around patient needs and existing endoscopy and bowel screening services. New patterns of working have been required to deliver BowelScope and challenges remain regarding adequate numbers of endoscopists.

Disclosure of Interest None Declared.

\section{PWE-057 A COMPOSITE MEASURE OF COLONIC INTUBATION (CIRC) IS BETTER ABLE TO DISTINGUISH PERFORMANCE OF COLONOSCOPY AND IS ASSOCIATED WITH HIGHER POLYP DETECTION RATES}

${ }^{1} \mathrm{R}$ Valori ${ }^{*},{ }^{2} S$ Damery, ${ }^{1} E$ Swarbrick, ${ }^{1} G$ Williams, ${ }^{1} \mathrm{~J}$ Anderson, ${ }^{1} \mathrm{M}$ Donnelly, ${ }^{1} \mathrm{D}$ Gavin. ${ }^{1} B S G / A C P G B I$, National Colonoscopy Audit Group; 'Department of Primary Care, Birmingham University, Birmingham, UK

10.1136/gutjnl-2014-307263.317

Introduction Caecal intubation rate (CIR) is a key performance indicator of colonoscopy. CIR with acceptable tolerance is only achieved in some patients with more sedation. As most colonoscopists achieve $>90 \%$ intubation, CIR alone cannot define excellence. This study proposes a more discerning, composite measure of colonoscopy intubation $\left(\mathrm{CIR}^{\mathrm{C}}\right)$ combining $\mathrm{CIR}$, patient comfort and sedation. Characteristics of patient, unit, equipment and colonoscopist associated with $\mathrm{CIR}^{\mathrm{C}}$, and the relation of $\mathrm{CIR}^{\mathrm{C}}$ to pathology detection are reported.

Methods Data from 20085 colonoscopies reported in the 2011 UK national audit (Gut 2013;62:242-249) were used for this analysis. The $\mathrm{CIR}^{\mathrm{C}}$ was defined as percentage of procedures achieving caecal intubation with median dose of midazolam or less, and nurse assessed comfort score of 1-3 (max 5). Multivariate analysis using binary logistic regression assessed patient, unit and colonoscopist factors in order to derive Odds Ratios (ORs) and $95 \%$ confidence intervals (CIs) for factors independently associated with $\mathrm{CIR}^{\mathrm{c}}$ after controlling for effects of all model variables. ORs have a significance level of $<0.001$ unless stated.

Results Overall achievement of $\mathrm{CIR}^{\mathrm{C}}$ was $54.1 \%$ ( $\mathrm{n}=10865$ procedures). The $\mathrm{CIR}^{\mathrm{C}}$ was better able to distinguish differences in performance than single measures such as CIR and polyp detection. Older age, male sex (OR 1.40; CI:1.32-1.49), adequate or excellent bowel prep and FOBT screen positive as indication were all associated with a higher chance of achieving $\mathrm{CIR}^{\mathrm{C}}$. Unit (JAG) accreditation (OR 1.26; CI 1.16-1.35) and the presence of one or more magnetic imagers in the unit (OR 1.29; 1.19-1.40) were associated with higher CIR $^{\mathrm{C}}$. Surgical speciality, greater annual volume, fewer years' experience and higher training/trainer status were associated with higher $\mathrm{CIR}^{\mathrm{C}}$. Achieving the $\mathrm{CIR}^{\mathrm{C}}$ was associated with significantly higher polyp detection rates (OR 1.12; 1.04-1.20) and higher cancer detection rates (OR 1.14; 0.98-1.32, $\mathrm{p}=0.10$ ).

Conclusion The $\mathrm{CIR}^{\mathrm{C}}$ provides a richer picture of colonoscopic intubation than CIR alone and is better able to discern factors associated with intubation competence in relation to patients, units, equipment and colonoscopists. Unit (JAG) accreditation was associated with higher $\mathrm{CIR}^{\mathrm{C}}$. Colonoscopists who perform more procedures, who have more experience of training or being trained, who have practised fewer years and who are in a surgical speciality had significantly higher rates of $\mathrm{CIR}^{\mathrm{C}}$. The $\mathrm{CIR}^{\mathrm{C}}$ was associated with a significantly higher polyp detection rate. It is proposed that $\mathrm{CIR}^{\mathrm{C}}$ replaces CIR as the key performance indicator for intubation of the colon.

Disclosure of Interest None Declared.

\section{PWE-058 INCREASING USE OF ENDOSCOPIC RESECTION, SINGLE-CENTRE EXPERIENCE}

S Ansari, R Sood*, V Hegade, L-A Smith. Digestive Diseases Centre, Bradford Royal Infirmary, Bradford, UK

\subsection{6/gutjnl-2014-307263.318}

Introduction Endoscopic Resection (ER), a technique, first pioneered in Japan for treating early gastric cancers, has gained 